\title{
Geophysical Research Letters
}

\section{RESEARCH LETTER}

10.1002/2016GL069915

\section{Key Points:}

- Analytic expression of seasonal flood frequency curve based on physically based parameters that embody information on daily flow dynamics - Daily flow regime (flow variability) controls the observed shape of flood frequency curves, including step changes for long return times

- The approach is suited to assess magnitude of events featured by return period longer than the sample size

Supporting Information:

- Supporting Information S1

- Figure S1

- Figure S2

- Figure S3

Correspondence to:

S. Basso,

stefano.basso@eawag.ch

\section{Citation:}

Basso, S., M. Schirmer, and G. Botter (2016), A physically based analytical model of flood frequency curves, Geophys. Res. Lett., 43, 9070-9076, doi:10.1002/2016GL069915.

Received 1 FEB 2016 Accepted 29 JUL 2016 Accepted article online 2 AUG 2016 Published online 7 SEP 2016

(O2016. American Geophysical Union. All Rights Reserved.

\section{A physically based analytical model of flood frequency curves}

\author{
S. Basso ${ }^{1,2}$, M. Schirmer ${ }^{1,2}$, and G. Botter ${ }^{3}$ \\ ${ }^{1}$ Department of Water Resources and Drinking Water, Eawag - Swiss Federal Institute of Aquatic Science and Technology, \\ Dübendorf, Switzerland, ${ }^{2}$ Centre for Hydrogeology and Geothermics, University of Neuchâtel, Neuchâtel, Switzerland, \\ ${ }^{3}$ Department ICEA and International Center for Hydrology “Dino Tonini”, University of Padova, Padua, Italy
}

Abstract Predicting magnitude and frequency of floods is a key issue in hydrology, with implications in many fields ranging from river science and geomorphology to the insurance industry. In this paper, a novel physically based approach is proposed to estimate the recurrence intervals of seasonal flow maxima. The method links the extremal distribution of streamflows to the stochastic dynamics of daily discharge, providing an analytical expression of the seasonal flood frequency curve. The parameters involved in the formulation embody climate and landscape attributes of the contributing catchment and can be estimated from daily rainfall and streamflow data. Only one parameter, which is linked to the antecedent wetness condition in the watershed, needs to be calibrated on the observed maxima. The performance of the method is discussed through a set of applications in four rivers featuring heterogeneous daily flow regimes. The model provides reliable estimates of seasonal maximum flows in different climatic settings and is able to capture diverse shapes of flood frequency curves emerging in erratic and persistent flow regimes. The proposed method exploits experimental information on the full range of discharges experienced by rivers. As a consequence, model performances do not deteriorate when the magnitude of events with return times longer than the available sample size is estimated. The approach provides a framework for the prediction of floods based on short data series of rainfall and daily streamflows that may be especially valuable in data scarce regions of the world.

\section{Introduction}

Assessing the magnitude and frequency of high flows in river basins is pivotal for many research and applied disciplines. Urban planning and water resources management, as well as the design of infrastructure, rely on dependable estimates of flood statistics. The assessment of economic viability and environmental sustainability of water facilities, which are experiencing a renaissance as engineering solutions to mitigate climate risk, requires characterizing the natural flooding regime of rivers [Poff et al., 2016]. A reliable assessment of high flow statistics is also central for the insurance industry [Horn and McShane, 2013] and for geomorphological research. Several studies have recently highlighted the relevance of the probability distribution of floods in setting long-term erosion rates of rivers [Rossi et al., 2016].

Flow extremes are traditionally estimated by using observations and probabilistic tools based on asymptotic statistical theories [Gnedenko, 1943; Coles, 2001]. Large data samples, however, are required to constrain statistical methods, especially those relating to the magnitude of rare events. Since long-term flow records are unavailable in most locations, tools have been developed which capitalize on different information sources (e.g., historical records, data from neighboring catchments, and improved process understanding) to characterize flood frequency curves [Merz and Blöschl, 2008]. Approaches that exploit the information content of daily flows [Claps and Laio, 2003] and methodologies that link high flow statistics to the catchment water balance [Sivapalan et al., 2005; Guo et al., 2014; Bartlett et al., 2015] have also been proposed to improve the reliability of model predictions.

In this paper, a physically based stochastic approach to estimate seasonal flood frequency curves is proposed. The method links the features of the extremal distribution of daily flows to a limited number of parameters embodying climate and landscape attributes, which are estimated from information about daily rainfall and discharge. The potential of the proposed method is illustrated through a set of applications in four catchments with heterogeneous hydroclimatic settings and flow regimes. The model performance in estimating the magnitude of events with return times longer than the available sample size is also discussed. 

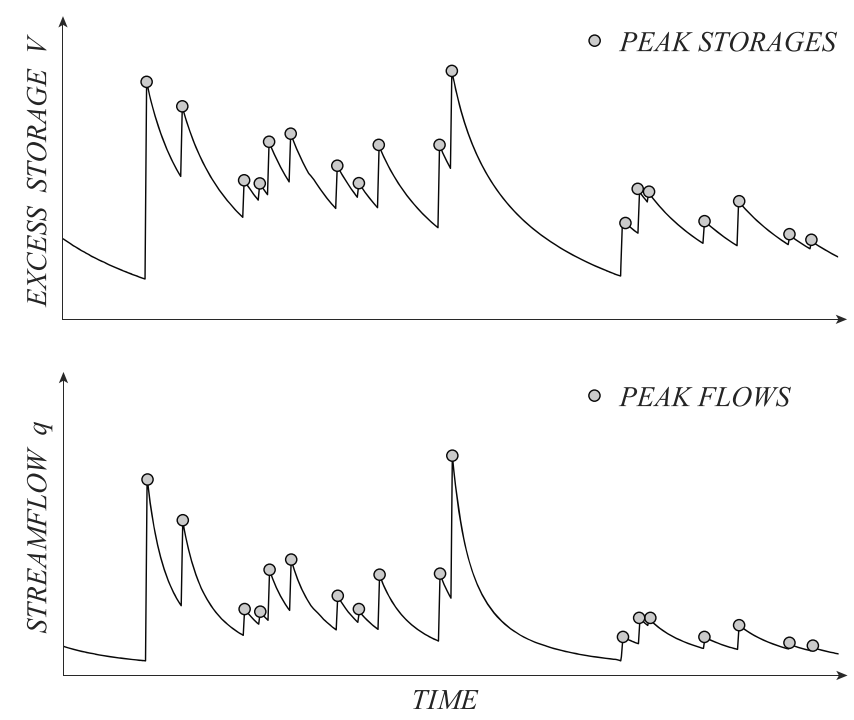

Figure 1. Excess storage $V$ and streamflow $q$ dynamics as produced by the mechanistic-stochastic model used in this study [Botter et al., 2009]. Flows (excess storages) occurring immediately after increments are termed peak flows (peak storages) and labeled with grey dots.

\section{Analytic Expression of the Seasonal Flood Frequency Curve}

The seasonal flood frequency curve is derived by building on a mechanistic-stochastic model of daily streamflows introduced by Botter et al. [2009]. Daily flow dynamics result from a catchment-scale balance of the soil moisture in the root zone [Laio et al., 2001] driven by stochastic increments due to infiltration from daily Poissonian rainfall (see supporting information) and evapotranspiration losses. When the rainfall pulses fill the deficit created by evapotranspiration and determine the exceedance of a critical soil moisture threshold (i.e., the water holding capacity of the soil), effective (i.e., streamflow producing) rainfall is triggered. This is modeled as a Poisson process with frequency $\lambda\left[T^{-1}\right]$ and exponentially distributed depths with average $\alpha[L]$. The effective rainfall recharges the excess storage of the catchment (i.e., the fraction of the catchment storage that can be drained) and eventually contributes to streamflow. In this framework, the dynamics of the specific (per unit catchment area) excess storage $V$ are driven by the following equation:

$$
\frac{\mathrm{dV}}{\mathrm{d} t}=-q+\xi(t)
$$

where $\xi(t)$ is a Poisson sequence of uncorrelated storage increases due to effective rainfall events and $q$ is a specific discharge. The latter is modeled through a deterministic relation between specific discharge and excess storage ( $q=\rho(V)$ ) [see Botter et al., 2009 and supporting information], which quantifies how the excess water is drained from the catchment and becomes streamflow. Here a power law storage-discharge relation is used, which allows the catchment drainage rate to vary as a function of the storage, thus implicitly incorporating the effect of different flow components (e.g., subsurface and surface runoff) [Basso et al., 2015]. Under the above assumptions, the probability density function (pdf) of the excess storage, $p(V)$, was derived analytically as a function of $\lambda, \alpha$ and the parameters of $\rho(V)$ (see Botter et al. [2009] and supporting information). The existence of a monotonic storage-discharge relation enables a formal link between the statistical features of $V$ and $q$. Excess storage dynamics described by equation (1) (and the corresponding discharge dynamics) are represented in Figure 1. The plot emphasizes that seasonal maxima of daily discharge belong to the subset of flows occurring immediately after effective rainfall events (grey dots in Figure 1), hereafter termed peak flows. Therefore, only the stochastic processes represented by the series of peak flows and peak storages (i.e., the excess storages right after streamflow jumps) are considered in the following derivations.

The probability distribution of peak storages, $p_{j}(V)$, is here obtained as a convolution between the pdf of the excess storage immediately before jumps and the pdf of the storage increments, which is an exponential distribution with average $\alpha$. Provided that the jump process is Markovian, the pdf of the excess storage before 
jumps is equal to the pdf of the excess storage itself, $p(V)$. By solving the convolution, the following expression for the probability distribution of peak storages is obtained:

$$
p_{j}(V)=C_{1} \exp \left(-\frac{V}{\alpha}\right) \exp \left[\frac{\lambda(2-a)}{(1-a)[K(2-a)]^{\frac{1}{2-a}}}\left(V-V_{0}\right)^{\frac{1-a}{2-a}}\right]
$$

where $C_{1}$ is a normalization constant, $a$ and $K$ are exponent and coefficient of the power law that defines the hydrograph recession in between flow-producing events, and $V_{0}$ represents a minimum or a maximum storage, depending on the storage-discharge relation chosen (see supporting information).

The probability distribution of peak flows is thus obtained by applying the derived distribution approach to equation (2) (see supporting information):

$$
p_{j}(q)=C_{2} q^{1-a} \exp \left[-\frac{q^{2-a}}{\alpha K(2-a)}+\frac{\lambda q^{1-a}}{K(1-a)}\right]
$$

where $C_{2}$ is a suitable normalization constant. The integration of equation (3) provides the nonexceedance cumulative probability of peak flows, $P_{j}(q)=\int_{0}^{q} p_{j}(q) \mathrm{d} q$.

In order to derive the probability distribution of seasonal maxima, let us assume a constant number $m$ of independent peak flows (see supporting information) in a given reference period $\tau$ (e.g., one season). Since peak flows are assumed to be independent, the probability that the maximum $q$ among the $m$ peak flows is not exceeded is the product of the probability of each peak flow to have a magnitude lower than $q$, i.e., $P_{j}\left[q_{1} \leq q\right] \cdots P_{j}\left[q_{m} \leq q\right]=P_{j}(q)^{m}$. Provided that the number of peak flows in the considered timespan is distributed according to a Poisson distribution with mean $\lambda \tau$ (because effective rainfall events are Poisson distributed), and recalling that $e^{z}=\sum_{m=0}^{+\infty} \frac{z^{m}}{m !}$ (with $z$ generic variable, equal to $\lambda \tau P_{j}(q)$ in this case), the following expression for the nonexceedance cumulative probability of seasonal maxima is obtained:

$$
P_{M}(q)=\sum_{m=0}^{+\infty} \frac{(\lambda \tau)^{m} e^{-\lambda \tau}}{m !} P_{j}(q)^{m}=\exp \left\{-\lambda \tau\left[1-P_{j}(q)\right]\right\}=\exp \left[-\lambda \tau D_{j}(q)\right]
$$

where $D_{j}(q)=1-P_{j}(q)$ represents the duration curve of peak flows.

The recurrence interval $T_{r}$ can be calculated as the inverse of the exceedance cumulative probability of flow maxima. Therefore, the following analytical expression of the seasonal flood frequency curve is obtained:

$$
T_{r}(q)=\frac{1}{1-P_{M}(q)}=\frac{1}{1-\exp \left[-\lambda \tau D_{j}(q)\right]}
$$

where $\lambda \tau$ represents the (dimensionless) average number of effective rainfall events during the considered season.

\section{Case Studies and Parameters Estimation}

The approach has been applied to four river basins belonging to different climatic regions of the central and eastern U.S. (see Figure 2a). The considered basins are the Sand Run (data from 1947 to 2014), a small $\left(\right.$ area $\left.=37 \mathrm{~km}^{2}\right)$ steep watershed in West Virginia; the Big Eau Plein River (1949-2014), a larger catchment $\left(\right.$ area $\left.=580 \mathrm{~km}^{2}\right)$ characterized by lower relief in Wisconsin; the Castor River (1953-1991 and 2001 -2012), a medium-sized basin (area $=1096 \mathrm{~km}^{2}$ ) in the rolling hills of Missouri; and the Redgate Creek (1962-2012), an ephemeral stream with an area of $45 \mathrm{~km}^{2}$ located in a semiarid region of Texas. The selected catchments encompass varied climatic and morphological conditions and are characterized by heterogeneous daily flow regimes, ranging from persistent to highly erratic [Botter et al., 2013]. The winter period in the Big Eau Plein River has been neglected, because the seasonal flow regime is affected by snow dynamics. Hence, an overall number of 15 seasonal flood frequency curves is used to illustrate main features and performance of the method.

The characterization of the seasonal flood frequency curve relies on specifying the four parameters $(\alpha, \lambda, a$, and $K$ ) of the mechanistic-stochastic model of daily streamflows (Table S1 in the supporting information). The estimation methods for $\alpha, \lambda$, and $a$ only employ daily rainfall and flow data and are detailed in Basso et al. [2015] and in the supporting information. The recession coefficient $K$, instead, is obtained by calibrating the analytic flood frequency curve on the observed seasonal maxima through maximum likelihood estimate. 
a) LOCATION OF CASE STUDIES
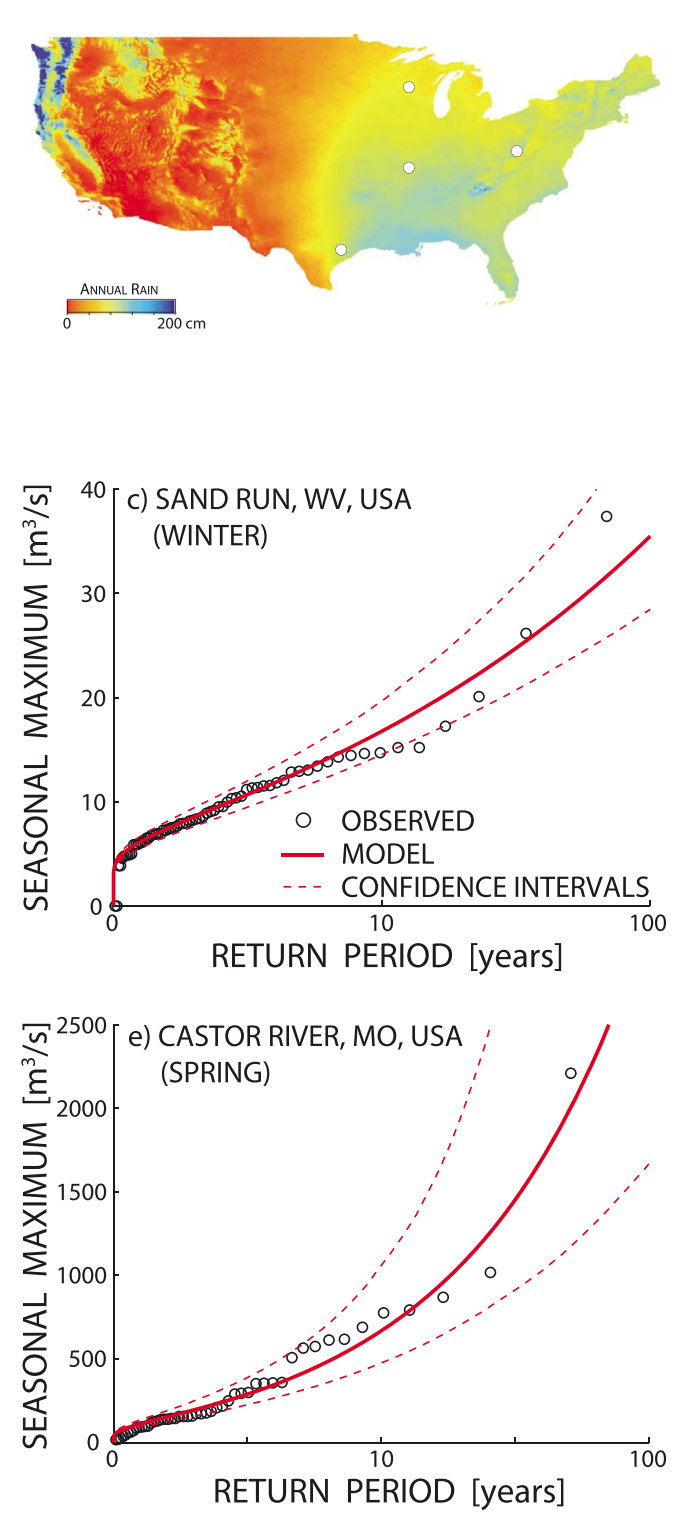
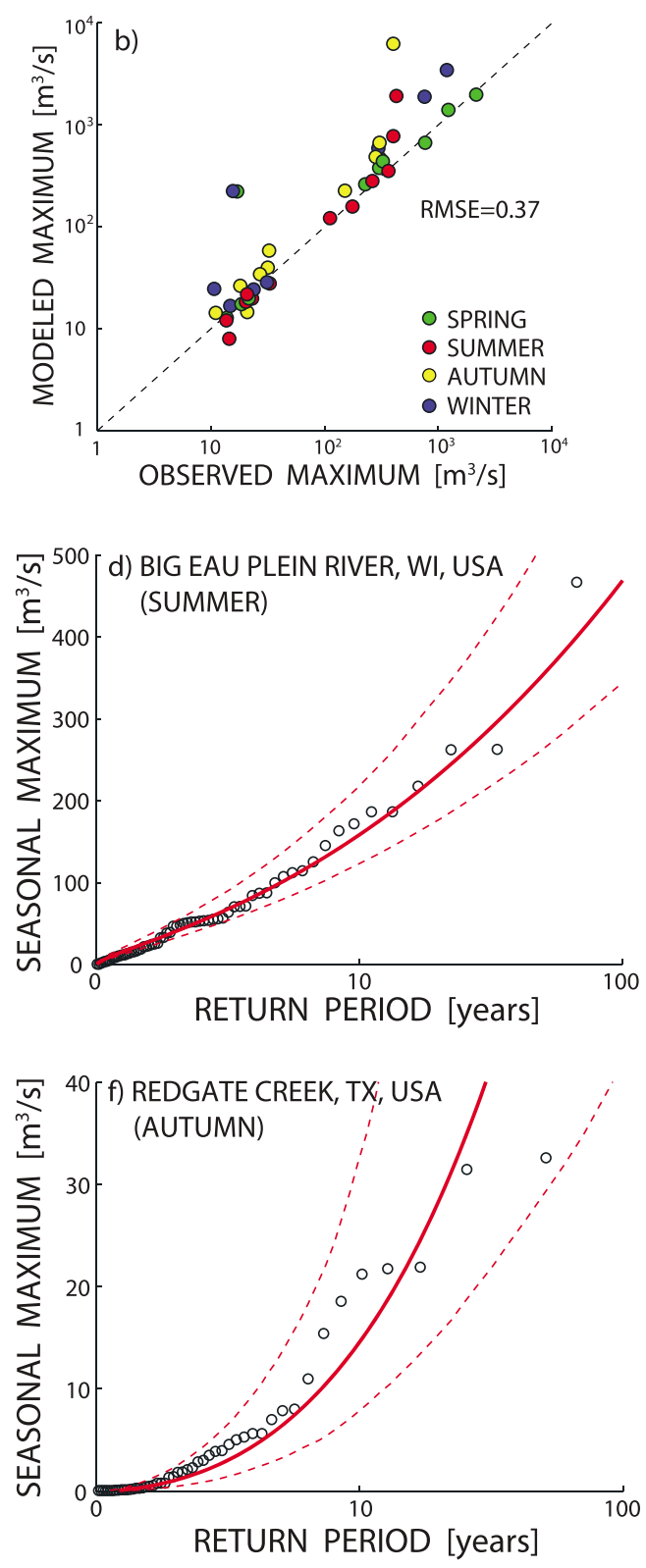

Figure 2. (a) Locations of the catchments outlets for the considered case studies. (b) Observed versus modeled seasonal maxima associated to $\operatorname{Tr}=10,30$, and 50 years for each catchment/season. The root-mean-square error (RMSE) of the estimates is reported. (c-f) Select seasonal flood frequency curves for the study catchments. For each case study, the season contributing the highest flow for the longest observed return period is displayed. Figures S1 and S2 show flood frequency curves for all case studies and seasons. Curves obtained from the entire series of observed data through Weibull plotting position are represented with dots, while solid red lines display estimates of the proposed analytical model. Confidence intervals of the model estimates are plotted with dotted red lines.

This is required by the pronounced sensitivity of high flows to antecedent catchment-scale soil moisture conditions (portrayed by the value of $K$ ) [Sivapalan et al., 2005; Shaw et al., 2013], which is further emphasized by focusing on maxima.

\section{Results}

Results of model application are displayed in Figure 2. Figure $2 \mathrm{~b}$ provides an overview of model performance by comparing, for all catchments and seasons, modeled flows with different return periods $\left(T_{r}=10,30\right.$, and 50 years) and the corresponding estimates obtained through Weibull plotting position of observed maxima. 


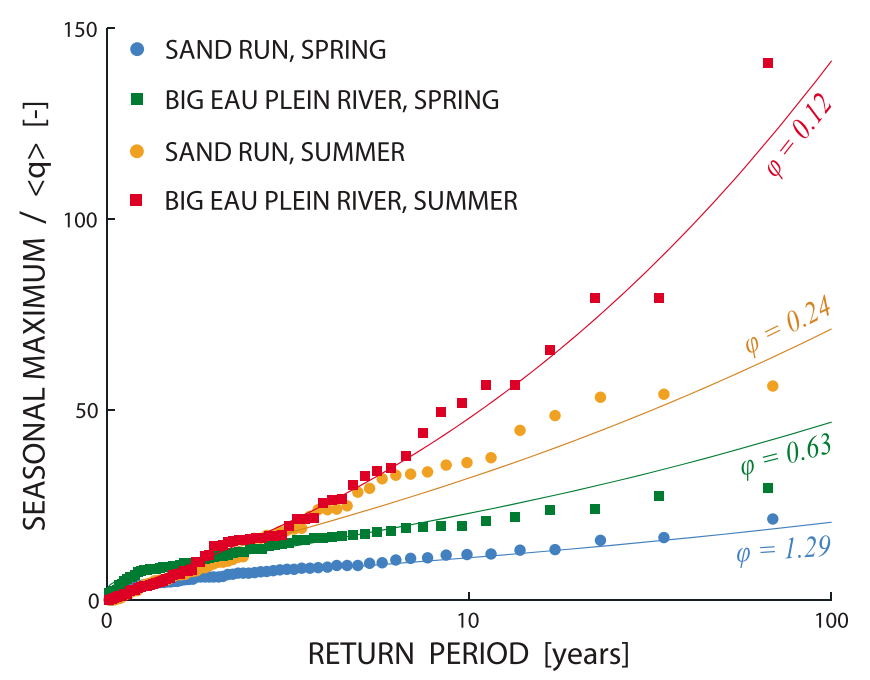

Figure 3. Normalized flood frequency curves (i.e., seasonal maximum divided by the average daily flow, $\langle q\rangle$ ) in four case studies characterized by decreasing persistency index $\phi$. For the calculation of the persistency index, $K$ has been estimated as in Basso et al. [2015]. Values of the coefficient of variation of daily flows associated to the four case studies are 1.30 (blue), 2.22 (green), 2.95 (yellow), and 4.13 (red). A decrease of the persistency index associated to the daily flow regime results in lower magnitude of events characterized by short return periods and higher magnitude of rare events in erratic regimes.

The model performs better for short and intermediate return periods, while bias appears for the highest $T_{r}$ (see also Figures S1 and S2). The root-mean-square error (RMSE) of the estimates is 0.37 , which is comparable with the performance of purely statistical methods (RMSE $=0.34$; see Figure S3). Figures $2 c-2 f$ compare the analytic flood frequency curves and the corresponding estimates from observations in all study catchments during selected seasons. The plots also show confidence intervals of model predictions (see supporting information). Although the performance varies for different catchments and seasons, estimates of the flood frequency curve obtained through Weibull plotting position of observed maxima (dots) fall in the predicted range of uncertainty. The observed diversity in the extent of the uncertainty across the study catchments may be explained by the dominant state of soil moisture prior to flood events in the analyzed catchments. In fact, higher predictability emerges from high values of the antecedent wetness, while lower soil moisture levels may enhance the uncertainty of the hydrologic response [Zehe and Blöschl, 2004].

The comparison among normalized flood frequency curves of Sand Run and Big Eau Plein River during different seasons (Figure 3) sheds light on the link between the shape of the flood frequency curve and the daily flow regime [see also Guo et al., 2014]. Flow regimes are here classified using the persistency index $\phi$, which represents the ratio between frequency of flow-producing events and mean recession rate. $\phi$ is inversely proportional to the variability of streamflows (see Figure 3 and Botter et al. [2013]), and can be expressed as a function of the model parameters as $\phi=\frac{\lambda}{K(\alpha \lambda)^{a-1}}$ (see supporting information). Figure 3 displays observed and modeled flood frequency curves corresponding to decreasing values of the persistency index, from 1.29 to 0.12. In persistent regimes (blue), the rise of the curve is intense for short return periods and then attenuates, with only a moderate increase of the magnitude of events associated to longer return periods. This produces concave flood frequency curves for a wide range of return periods. On the contrary, for erratic regimes (yellow and red) the flood frequency curve becomes convex for moderate values of $T_{r}$, which implies a sharp increase of the seasonal maxima (step change, sensu Rogger et al. [2012]) for long return periods. The increasing erraticity of the flow regime (from blue to red) is associated with a more pronounced change of concavity of the flood frequency curves, with step changes emerging in more erratic regimes. The observed link between the shape of the flood frequency curve and the underlying persistency index provides a formal relation between the behavior of the extremes and a set of climatic features $(\lambda, \alpha)$ and soil and geomorphic attributes $(a, K)$ of the catchment [Doulatyari et al., 2015], which are summarized by the value of $\phi$.

Figure 4 illustrates the reliability of the proposed model with respect to decreased lengths of the available data series. For each season, sliding windows with fixed length of 20,10, or 5 years are applied to the two longest time series available (for the Sand Run and the Big Eau Plein River) to generate shorter samples. These samples 

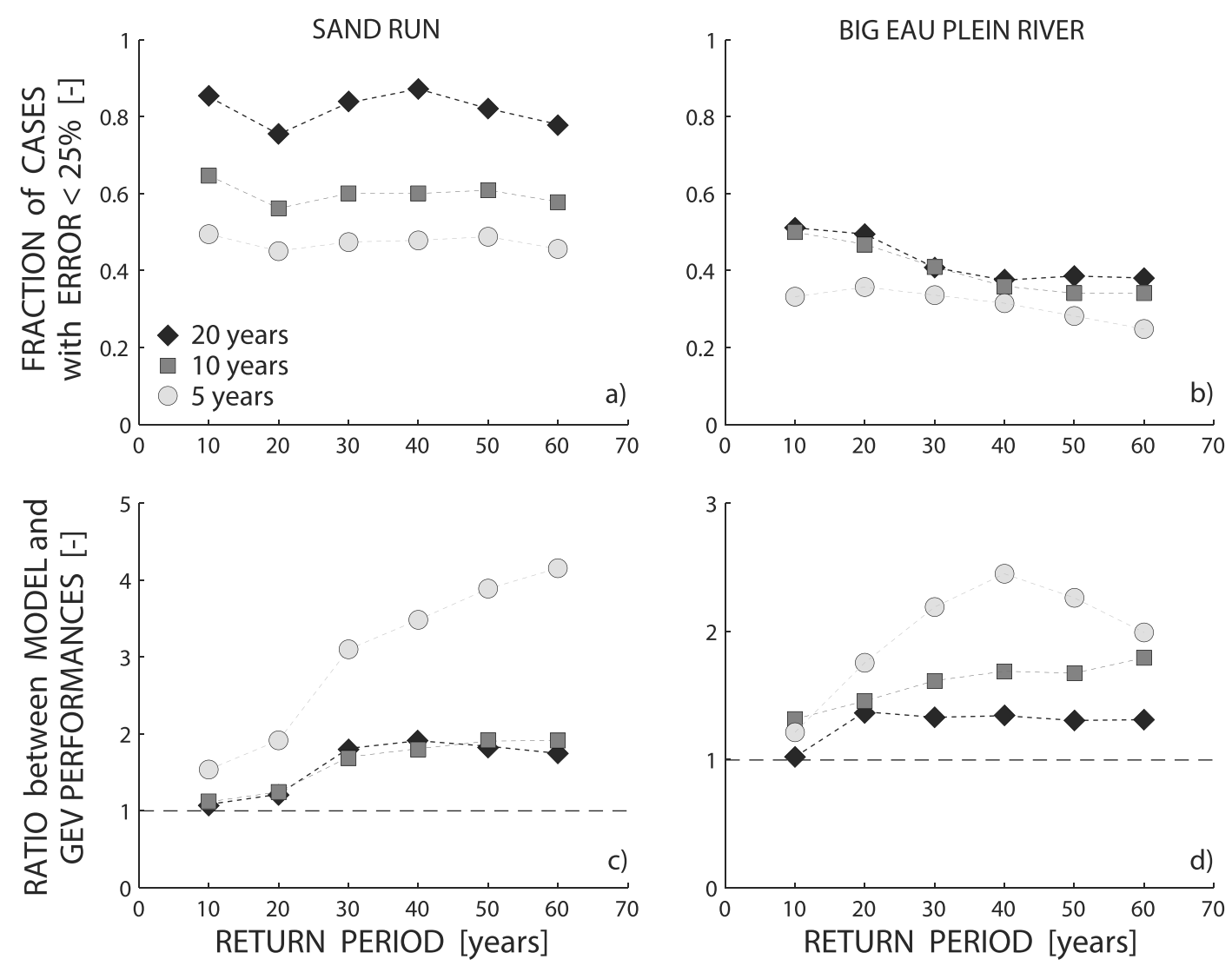

Figure 4. $(a, b)$ Performance of the proposed analytical model in predicting flood frequency curves with short data series. The longest available time series, which ensure more robust estimates of flood frequency curves from observed data, have been selected for the analysis. Samples of 20, 10, and 5 years drawn from the entire data set through a moving window are used to estimate analytic seasonal flood frequency curves. Model results are then compared with those obtained from the complete data series through Weibull plotting position. Errors are computed for a set of return periods (from 10 to 60 years). The plot shows the fraction of cases displaying an error $(\epsilon)$ lower than $25 \%$. The shorter the number of years (seasons) of available data, the lower the fraction of cases displaying $\epsilon<25 \%$. Performances are instead quite constant for increasing return periods. The same behavior is found by setting different thresholds for the acceptable error. (c, d) Comparison between performances of the proposed analytical model and of generalized extreme value (GEV) distributions calibrated through maximum likelihood on the same samples. The ratio between results shown in Figures $4 \mathrm{a}$ and $4 \mathrm{~b}$ and the equivalent metrics computed for GEV distributions is displayed. Performances are comparable for long data series available (e.g., 20 years) and short return periods (e.g., 10 years). The proposed method exhibits better performances for rare events (high return periods), particularly when only short observed data series (e.g., 5 years) are available to constrain the models. In all plots higher plotting levels indicate better performances of the proposed model.

are used to constrain the model, and its predictions for a set of return periods ranging from 10 to 60 years are compared with the estimates obtained from the entire dataset available through Weibull plotting position. The percent error $(\epsilon)$ between model and data based estimates is computed each time. Figures $4 \mathrm{a}$ and $4 \mathrm{~b}$ represent, for each return period, the fraction of cases providing errors lower than 25\% (arbitrarily assumed as an acceptable error threshold). As expected, the fraction of cases characterized by $\epsilon<25 \%$ diminishes with decreasing length of the sample used for calibration. The fraction of acceptable cases is lower in the erratic than in the persistent regime. This is due to the higher streamflow variability of erratic regimes, which makes them intrinsically unpredictable [Botter et al., 2013]. Interestingly, the performance is quite stable with increasing return periods. This result hints to a structural robustness of the model, whose prediction weakly depends on the specific flood events (and return periods) sampled in the observed data series used for calibration.

In order to better appreciate the significance of these results, Figures $4 \mathrm{c}$ and $4 \mathrm{~d}$ compare the performance of the proposed approach with that of a generalized extreme value distribution whose parameters are calibrated by maximum likelihood estimate on the same samples used for the calibration of the physically based approach. The plots evidence systematically higher performances of the proposed method for return periods 
longer than the sample size. These performances stem from the model's capability to exploit experimental information on the full range of discharges experienced by rivers.

The proposed framework may prove particularly useful to estimate the magnitude of events with long recurrence interval (as required, e.g., to evaluate long-term sediment delivery [Kirchner et al., 2001] and erosion rates in bedrock rivers [Lague et al., 2005]), or when only short records are available, for example, due to temporary or newly installed flow gauges. This is the case for the majority of the catchments worldwide [Müller and Thompson, 2015].

\section{Conclusions}

A novel physically based analytic expression of the seasonal flood frequency curve is presented. The expression is grounded on a mechanistic-stochastic model of daily streamflow dynamics, whose parameters embody hydroclimatic and landscape attributes of the contributing catchment. Only one parameter related to the antecedent wetness state of the catchment needs to be fitted on observed flow maxima. The application of the method in four rivers featuring heterogeneous flow regimes (from persistent to highly erratic) is discussed. The model provides reliable estimates of the flows associated to a set of return periods and captures the diverse shapes of flood frequency curves emerging in erratic and persistent regimes. Thanks to the structure of the proposed method, which uses information on the whole range of flows experienced by rivers, model performances do not deteriorate significantly when flows characterized by return times longer than the available sample size are estimated. The proposed framework is a first step toward the prediction of high flow statistics based on climate and landscape attributes and may be especially worth in data scarce regions of the world.

Acknowledgments

The U.S. Geological Survey (http://waterdata.usgs.gov) and the National Climatic Data Center (http://cdo.ncdc.noaa.gov/) are acknowledged for providing hydrologic and climatic data. This study was funded by the Swiss National Science Foundation (SNF, project 200021-149126). Additional support was provided by the Competence Center Environment and Sustainability (CCES) of the ETH domain in the framework of the RECORD Catchment project. The first author acknowledges a SNF Mobility Fellowship. The Marani Lab at Duke University, Durham, NC, USA, is acknowledged for providing computational resources. Roberto Corvaja and Nicola Pozzobon are gratefully acknowledged for the useful discussions on mathematical derivations.

\section{References}

Bartlett, M. S., E. Daly, J. J. McDonnell, A. J. Parolari, and A. Porporato (2015), Stochastic rainfall-runoff model with explicit soil moisture dynamics, Proc. R. Soc. A, 471, 20150389, doi:10.1098/rspa.2015.0389.

Basso, S., M. Schirmer, and G. Botter (2015), On the emergence of heavy-tailed streamflow distributions, Adv. Water Resour., 82, 98-105, doi:10.1016/j.advwatres.2015.04.013.

Botter, G., A. Porporato, I. Rodriguez-Iturbe, and A. Rinaldo (2009), Nonlinear storage-discharge relations and catchment streamflow regimes, Water Resour. Res., 45, W10427, doi:10.1029/2008WR007658.

Botter, G., S. Basso, I. Rodriguez-Iturbe, and A. Rinaldo (2013), Resilience of river flow regimes, Proc. Natl. Acad. Sci. U.S.A., 110(32), 12,925-12,930, doi:10.1073/pnas.1311920110.

Claps, P., and F. Laio (2003), Can continuous streamflow data support flood frequency analysis? An alternative to the partial duration series approach, Water Resour. Res., 39(8), 1216, doi:10.1029/2002WR001868.

Coles, S. (2001), An Introduction to Statistical Modeling of Extreme Values, Springer, London, U. K.

Doulatyari, B., A. Betterle, S. Basso, B. Biswal, M. Schirmer, and G. Botter (2015), Predicting streamflow distributions and flow duration curves from landscape and climate, Adv. Water Resour., 83, 285-298, doi:10.1016/j.advwatres.2015.06.013.

Gnedenko, B. (1943), Sur la distribution limite du terme maximum d'une serie aleatoire, Ann. Math., 44(3), 423-453, doi:10.2307/1968974.

Guo, J., H.-Y. Li, L. R. Leung, S. Guo, P. Liu, and M. Sivapalan (2014), Links between flood frequency and annual water balance behaviors: A basis for similarity and regionalization, Water Resour. Res., 50, 937-953, doi:10.1002/2013WR014374.

Horn, D., and M. McShane (2013), Flooding the market, Nat. Clim. Change, 3, 945-947, doi:10.1038/nclimate2025.

Kirchner, J. W., R. C. Finkel, C. S. Riebe, D. E. Granger, J. L. Clayton, J. G. King, and W. F. Megahan (2001), Mountain erosion over 10 yr, 10 k.y., and 10 m.y. time scales, Geology, 29, 591-594.

Lague, D., N. Hovius, and P. Davy (2005), Discharge, discharge variability, and the bedrock channel profile, J. Geophys. Res., 110, F04006, doi:10.1029/2004JF000259.

Laio, F., A. Porporato, L. Ridolfi, and I. Rodriguez-Iturbe (2001), Plants in water-controlled ecosystems: Active role in hydrologic processes and response to water stress: II. Probabilistic soil moisture dynamics, Adv. Water Resour., 24(7), 707-723, doi:10.1016/S0309-1708(01)00005-7.

Merz, R., and G. Blöschl (2008), Flood frequency hydrology: 1. Temporal, spatial, and causal expansion of information, Water Resour. Res., 44, W08432, doi:10.1029/2007WR006744.

Müller, M. F., and S. E. Thompson (2015), Stochastic or statistic? Comparing flow duration curve models in ungauged basins and changing climates, Hydrol. Earth Syst. Sci. Discuss., 12, 9765-9811, doi:10.5194/hessd-12-9765-2015.

Poff, N. L., et al. (2016), Sustainable water management under future uncertainty with eco-engineering decision scaling, Nat. Clim. Change, 6, 25-34, doi:10.1038/nclimate2765.

Rogger, M., H. Pirkl, A. Viglione, J. Komma, B. Kohl, R. Kirnbauer, R. Merz, and G. Blöschl (2012), Step changes in the flood frequency curve: Process controls, Water Resour. Res., 48, W05544, doi:10.1029/2011WR011187.

Rossi, M. W., K. X. Whipple, and E. R. Vivoni (2016), Precipitation and evapotranspiration controls on daily runoff variability in the contiguous United States and Puerto Rico, J. Geophys. Res. Earth Surf., 121, 128-145, doi:10.1002/2015JF003446.

Shaw, S. B., T. M. McHardy, and S. J. Riha (2013), Evaluating the influence of watershed moisture storage on variations in base flow recession rates during prolonged rain-free periods in medium-sized catchments in New York and Illinois, USA, Water Resour. Res., 49, 6022-6028, doi:10.1002/wrcr.20507.

Sivapalan, M., G. Blöschl, R. Merz, and D. Gutknecht (2005), Linking flood frequency to long-term water balance: Incorporating effects of seasonality, Water Resour. Res., 41, W06012, doi:10.1029/2004WR003439.

Zehe, E., and G. Blöschl (2004), Predictability of hydrologic response at the plot and catchment scales: Role of initial conditions, Water Resour. Res., 40, W10202, doi:10.1029/2003WR002869. 